\title{
PATHWISE CONNECTIVITY OF THE SPATIAL NUMERICAL RANGE
}

\author{
TOFIK Y. KULIYEV
}

(Communicated by Palle E. T. Jorgensen)

\begin{abstract}
In this paper we prove that the spatial numerical range of a given operator on a separable Banach space is pathwise connected.
\end{abstract}

In 1977 Luna [1] gave a partial answer to a question of Bonsall and Duncan [2] by proving that the spatial numerical range of a bounded linear operator is pathwise connected in the case of a reflexive Banach space. Later in 1985 Weigel [3] simplified considerably Luna's proof. In the present paper we give a nearly elementary proof of the same fact in the case of a separable Banach space.

Let $E$ be a complex Banach space, and let $E^{*}$ be the conjugate space of $E$. We set, for $x \in E$,

$$
D(x)=\left\{y \in E^{*} ;\|y\|=\|x\| \text { and } y(x)=\|x\|^{2}\right\} .
$$

The set-valued mapping $x \mapsto D(x)$ is usually called the duality mapping of $E$. The set $D(x)$ is a nonempty convex and $w^{*}$-compact subset of $E^{*}$. The graph of $D$ is defined to be the set

$$
\Gamma=\Gamma_{E}=\{(x, y) ; y \in D(x)\} .
$$

Theorem 1. Let $E$ be a separable Banach space. Then the graph $\Gamma_{E}$ of the duality mapping of $E$ is a pathwise connected set.

Proof. Let $\left(x_{1}, y_{1}\right),\left(x_{2}, y_{2}\right) \in \Gamma$. Without real loss of generality we may assume that $\left\|x_{1}\right\|=\left\|x_{2}\right\|=1$. Let $V=\bigvee\left\{x_{1}, x_{2}\right\}$ be the subspace of $E$ generated by $x_{1}, x_{2}$, and let $s(X)$ be the unit sphere of a Banach space $X$. It is sufficient to prove that the set $G=\{(x, y) ; x \in s(V), y \in D(x)\}$ is pathwise connected.

Claim 1. $G$ is compact. Let $\left(\left(x_{i}, y_{i}\right)\right)_{i \in I}$ be a net in $G$. There is a subnet $J$ of $I$ such that $\left(x_{j}\right)_{j \in J}$ converges in norm to some element $x$. We can choose a subnet $K$ of $J$ for which $\left(y_{k}\right)_{k \in K}$ converges in $w^{*}$-topology to some $y$. We must show that $y \in D(x)$. But we have

$$
0 \leq\|y(x)-1\| \leq\left\|y(x)-y_{k}(x)\right\|+\left\|y_{k}(x)-y_{k}\left(x_{k}\right)\right\| \rightarrow 0 .
$$

Received by the editors April 1, 1993.

1991 Mathematics Subject Classification. Primary 47A12; Secondary 46B20.

Key words and phrases. Numerical range, duality mapping, pathwise connectivity. 
Claim 2. $G$ is connected. This can be proved as in [2].

Claim 3. $G$ is a matrizable continuum. Since $E$ is separable, the $w^{*}$ topology on $s\left(E^{*}\right)$ is metrizable; hence, our statement follows from the Claims 1 and 2.

Claim 4. $G$ is locally connected. Note that $s(V)$ is locally connected. Indeed, since $\operatorname{dim} V<\infty, s(V)$ is homeomorphic to the unit sphere of a finitedimensional Hilbert space, which is, obviously, locally connected. Now let $(x, y) \in G$ and $U$ be a neighbourhood of $(x, y)$. Then $U \supset\left(U_{1} \times U_{2}\right) \cap G$, where $U_{1}$ is a neighbourhood of $x$ and

$$
U_{2}=\left\{y^{\prime} \in E^{*} ;\left|\left(y^{\prime}-y\right)\left(x_{n}\right)\right|<\varepsilon\right\},
$$

$x_{n} \in E, n=1,2, \ldots, m$, is a convex neighbourhood of $y$. Since $s(V)$ is locally connected and connected, the set of closed connected neigbourhoods of $x$ forms a base at the point $x$, so we may assume that $U_{1}$ is a closed connected set. But then $\left(U_{1} \times U_{2}\right) \cap G$ is connected, which can be proved in the same way as in [2].

Claim 5. $G$ is pathwise connected. This follows from Claims 3 and 4 and $[4,6.3 .11]$.

The proof is completed.

We do not know whether it is possible to omit the separability condition.

As a corollary we get the following:

Theorem 2. Let $E$ be a separable Banach space, and let $T$ be a bounded linear operator on $E$. Then the spatial numerical range of $T$, i.e., the set

$$
W(T)=\{y(T x) ;\|x\|=\|y\|=y(x)=1\},
$$

is pathwise connected.

\section{REFERENCES}

1. G. Luna, On the numerical range in reflexive Banach spaces, Math. Ann. 231 (1977), 33-38.

2. F. F. Bonsall and J. Duncan, Numerical ranges of operators on normed spaces and of elements of normed algebras, London Math. Soc. Lecture Note Ser., vol. 2, Cambridge Univ. Press, Cambridge, 1971.

3. H. Weigel, Reflexivity and numerical range, Exposition. Math. 3 (1985), 373-374.

4. R. Engelking, General topology, Heldermann, Berlin, 1989.

1385 Pine St. \#21, San Francisco, California 94109

Current address: Department of Mathematics, University of California at Berkeley, Berkeley, California 94720

E-mail address: kuliyev@math.berkeley.edu 